\title{
Optimisation of Biological Transport Networks
}

\author{
Yuankai Lu and Dan $\mathrm{Hu}^{*}$ \\ School of Mathematical Sciences, Institute of Natural Sciences, and MOE-LSC, \\ Shanghai Jiao Tong University, Shanghai 200240, China.
}

Received 18 May 2021; Accepted (in revised version) 13 July 2021.

\begin{abstract}
Transport networks such as blood vessel systems and leaf venation are universally required for large-size living organisms in order to overcome the low efficiency of the diffusion in large scale mass transportation. Despite substantial differences in living organisms, such networks have many common patterns - viz. biological transport networks are made up of tubes and flows in tubes deliver target substances. Besides, these networks maintain a tree-like backbone attached with small loops. Experimental and mathematical studies show many similarities in biological mechanisms, which drive structural optimisation in biological transport networks. It is worth noting that the structural optimisation of transport networks in living organisms is achieved in the sense of energy cost as a consequence of natural selection. In this review, we recall the exploration history and show mathematical structures used in the design of biological transport networks.
\end{abstract}

AMS subject classifications: 37N25, 76D55

Key words: Biological transport networks, blood vessels, optimisation principle, adaptation.

\section{Introduction}

Transport networks play important role in natural and industrial systems. Rivers carry water to oceans and lakes, highways and railways transport people and goods all around the world, telecommunications networks transmit information, and blood vessels and leaf veins transport substances in living organisms.

However, the transport capacity of various networks is limited by the flow processing capacity of network nodes (aviation networks), by the conductance and width of network edges (rivers and blood circulation systems), or by both factors mentioned (internet). In general, the conservation of matter leads to constraints on the flow in different edges. If there is no node capacity, the flow constraints are instantaneously satisfied. Otherwise, a delay is allowed to satisfy the flow constraints. For information networks, such constraints are weakened greatly by a possible information replication on nodes. The limitations in transport capacity and constraints in flows determine the main geometrical and topological

*Corresponding author. Email address: hudan80@sjtu.edu.cn (D. Hu) 
characteristics of the networks - e.g. biological transport networks usually contain various loops while maintaining an impression of a tree-like backbone [23, 29]. Here, we review the exploration of the designing principle of biological transport networks.

The first breakthrough in biological transport networks was the experimental finding of an approximate cubic law in blood vessel bifurcation - viz. $D^{3}=D_{l}^{3}+D_{r}^{3}$, where $D, D_{l}$, and $D_{r}$ are the radii of the parent vessel and its left and right daughter vessels, respectively [71]. This law is now referred to as Murray's law due to Murray's deep insight between the bifurcation relation and the optimisation principle in energy cost. According to Murray explanations, this is a consequence of another cubic relation between blood flow $Q$ and vessel radius $D, Q \propto D^{3}$. In Murray's theory, the latter cubic relation is obtained by the optimisation of the total energy cost - i.e. the fluidic cost in driving blood flow in the vessel and the biological cost in metabolism and material, which includes the material cost in producing blood cells and building the blood vessel wall and the energy consumption in functioning of these cells [54]. Murray's law is also observed in plant vessels and leaf venation [49].

Murray's law provides the deep understanding of the local structure of biological networks. Following the idea of energy-cost optimisation, mathematical models have also been used to understand the geometrical and topological characteristics of an entire biological transport network. For different transport networks, a power law relation $E_{m}=C^{\gamma}$ can be generally introduced for the metabolic and material cost $E_{m}$ and flow resistance $C$. This relation is used to show that for fixed flow sources (sinks) a network has a tree-like structure if $\gamma<1[5,6,14]$. In general, there are many loops attached to the tree-like backbone in biological transport networks $[9,11,29,34,55]$. Animals and plants can benefit from such loop structures in various ways. By incorporating the risk tolerance in network damages [29] or the effects of fluctuating flow distributions [9, 29, 30], optimal structures are shown to be loopy networks while maintaining a major structure of a tree-like backbone. Such a structure is also believed to afford great benefits to living systems for their mechanical robustness [34].

Optimisation of the energy cost can be viewed as the consequence of natural selection. Highly efficient and robust transport networks, which optimise the energy cost while satisfying tissue demanding, bring tremendous competitive advantages to species. Nevertheless, in order to achieve such an optimisation, life systems have to find special mechanisms such as an adaptation dynamics driven by specific stimuli (which means signals sensed by cells and modulate their cellular dynamics).

The Murray's law also suggests that the wall shear stress, which is proportional to $Q / D^{3}$, is a constant in the entire circulation system at optimal state. This implies that the wall shear stress should be an important stimulus that drives blood vessel adaptation to achieve the optimisation of the network structure. Indeed, experimental studies verified that the wall shear stress in a circulation system lies in a relatively narrow range $[19,23,25,58-$ 60]. Further studies have shown that endothelial cells, which form the inner layer blood vessel walls, can really sense the wall shear stress [31-33]. The wall shear stress acts as a key stimulus for both blood flow regulation in the short term response and blood vessel adaptation in the long term response $[19,23,25,31-33,58-60]$. 
Mathematical models are build up to describe the adaptation of the vessel diameter in response to wall shear stress $[19,23,25,59]$. It is interesting to not that the parallel vessel systems where two or more vessels start from the same point and converge to different points, are unstable in such kind of adaptation dynamics $[19,25]$. More precisely, the diameter of one of two parallel vessels converges to zero as time tends to infinity. One may worry about the adaptation stability of arterial trees, which are very similar to parallel systems since the blood pressures at the inlets of capillaries are maintained close to a fixed value [25]. In fact, by incorporating the short term response of blood flow regulation, the adaptation of arterial trees becomes stable [25].

The two research lines of structural optimisation and vessel adaptation merge back again in Cai-Hu's adaptation model and later adaptation models for biological transport networks $[23,61,62]$. This adaptation model is driven by local stimuli, mainly the second moment of the wall shear stress. This adaptation dynamics can be regarded as a gradient flow to optimise the total energy cost for both fixed and fluctuation flow distribution. In particular, a number of loops in the network can be stabilised for sufficiently strong fluctuation in flow distribution. The adaptation model is successfully used in predicting vessel pruning (which means disappearance of the existing blood vessels) observed in embryo zebra-fish [8]. This success shows that the adaptation in response to the wall shear stress is indeed employed by animals to optimise the efficiency of their circulation system. Pruning of unnecessary blood vessels as a sequent of vessel adaptation has been shown to be a significant means in optimising the network structures $[8,23,66]$.

With a pre-given network, the optimisation and adaptation models can provide an optimal network. Nevertheless, how biological transport networks are initially formed remains yet to be answered. Active explorations have also been undertaken to the mechanisms for emergence of biological transport networks. The importance of hormone transport has been shown experimentally in the initiation process of biological transport networks in both animals and plants. For embryo vasculogenesis [26], self aggregation of endothelial cells leads to the formation of the earliest circulation system before the heart starts to beat. Agent based modeling and numerical studies suggest the essentiality of migration and elongation of endothelial cells in vasculogenesis and angiogenesis [35, 51]. For leaf budding $[4,11,48,69]$, cell differentiation is induced along concentrated pathways for delivery of auxin, which is an important plant hormone for growth and cell differentiation. Cells on this pathway eventually develop to form the leaf venation. Canalisation, a gene regulation model for cell differentiation stimulated by auxin, was hypothesized in the formation of leaf veins as a response to auxin delivery [11,17, 48, 52, 53, 63-65].

Although the above agent based models have been successful in relating the signals of hormone transport with cell differentiation and emergence of vascular networks, they are silent in the optimisation of network structures. Cai and Hu developed a continuum model for the initiation process of biological transport networks [24] such that initiation and adaptation processes are integrated in a single model. By coupling the hormone transport and the adaptation process, this model naturally optimises the total energy cost, including the energy cost in transportation and metabolic and material costs. In this model the energy cost function decreases continuously and drives the canalisation. As the result, optimal 
structures are obtained as the steady state of the evolution dynamics. The new mathematical structure in the Cai-Hu continuum model has attracted widespread attention and interest. A series of work has been done on the well-posedness [2, 20,21, 38-41, 43, 68, 72] and numerical methods $[1,16,22]$ for the model. It should be noted that some of the works appeared before the Cai-Hu model was published and just make references to their lectures.

Despite the widespread success, more efforts are needed in biological transport network studies. First, the optimisation and adaptation models of biological transport networks must be integrated into cellular biological processes. It remains unclear how the microscopic cellular dynamics is connected with the macroscopic adaptation. Second, the adaptation of vessel segments plays an important role in angiogenesis and root development. However, it is still far from a clear picture on what stimuli help to maintain the stability of capillary networks while meeting the needs of the tissue, and efficient numerical methods are extremely important in simulations of such large scale systems [44]. Finally, new mathematical structures of biological transport networks may provide new insights and tools for applications in other fields, such as graph theory and matrix optimisation. It also allows further applications on designing efficient network for transmission of mass, energy, and information [27]. Studies on the models can also shed lights on the optimisation of transport networks with different cost functions and different constraints. The models can also be used in the design of artificial blood vessel networks [46]. From this point of view, further studies are important in discovering the properties and exploring the applications of the discrete and continuum models. This review aims to provide a complete picture on the state of research and promote interdisciplinary studies on biological transport networks.

\section{Necessity of Biological Transport Networks}

Typically, the diffusion coefficient $D_{0}$ of a molecule to move within its local tissue environment is of the order $10 \sim 1000 \mu \mathrm{m}^{2} / \mathrm{s}$ [66]. From a simple dimensional analysis, we can estimate the time $T_{0} \sim L^{2} / D_{0}$ required for a molecule to traverse a given distance $L$. This quadratic dependence indicates the inefficiency of the diffusion for mass delivery in organisms with large body size. Biological transport networks are designed to overcome this inefficiency of the diffusion for large scale transport. It is interesting to see that similar network structures appear in silicon-on-insulator (SOI) circuits for heat removal due to a similar quadratic dependence for heat conduction $[7,57]$.

Biological transport networks consist of hollow tubes. Flows in tubes are used to deliver target molecules - e.g. oxygen, carbon dioxide, and water molecules. In this way, convection is used for efficient mass transport. Diffusion is used for transport only between the tissue and terminal branches of the transport networks, such as capillaries, hairy roots, and terminal leaf veins. A typical spacing between neighboring capillaries and between terminal leaf veins is of the order $100 \mu m[44,66]$. On this spacial scale, diffusion is sufficiently efficient for the time scale of living organisms.

\subsection{Kirchhoff's equation for biological transport networks}

A blood vessel can be regarded as a cylindrical tube, whereas the leaf vein is usually 
formed by a bundle of small tubes. The blood flow in large blood vessels can be very complex, which can indeed affect the geometry (vessel diameter and vessel wall thickness) of blood vessels $[59,60]$. In this review, we discard this complex effect and consider only small vessels.

For fluids in small tubes - e.g. with luminal diameters smaller than $0.6 \mathrm{~mm}$, the Reynolds number is sufficiently low and the flow in the tube can be well approximated by the Poiseuille flow $[19,59,60]$. More exactly the fluid velocity along the cylindrical tube satisfies

$$
v(r)=v_{0}\left(1-\left(\frac{r}{R_{0}}\right)^{2}\right)
$$

where $v_{0}$ is the maximal velocity at the center of the cross section, $r$ the polar radius, and $R_{0}$ the vessel radius. In this case, the volumetric flow rate $Q=2 \pi \int_{0}^{R_{0}} v(r) r d r$ in the tube and the wall shear stress $\tau_{w}=\left.\mu \frac{\partial v}{\partial r}\right|_{r=R_{0}}$ on the wall is determined by the force balance (note that the inertial force is negligible when the Reynolds number is small)

$$
Q=\frac{\triangle P \pi D^{4}}{128 \mu L}, \quad \tau_{w}=\frac{32 \mu Q}{\pi D^{3}}=\frac{\triangle P D}{4 L},
$$

where $\mu$ is the viscosity of the fluid, $\triangle P$ the pressure drop, $L$ the tube length, and $D=2 R_{0}$ the tube diameter. The resistance $R$ of a vessel, such as a blood vessel or a leaf vein and its reciprocal, the conductance $C$, are defined as

$$
R=\frac{\triangle P}{n Q}=\frac{128 \mu L}{\pi n D^{4}}, \quad C=\frac{\pi n D^{4}}{128 \mu L}
$$

where $n$ is the number of parallel tubes in the vessel. For blood vessels, $n$ is usually equal to 1 , whereas for vessels in plants such as leaf veins, $n$ is greater than 1 except for the terminal branches of the networks. The conductance depends only on the geometry of the vessel. It is very sensitive to the diameter change due to the quartic power in the Eq. (2.2). In fact, this sensitivity is artfully employed by blood vessel systems by accurately controlling the luminal diameter via contraction and dilation of smooth muscle cells [31-33].

The flow distribution in biological transport networks is determined by the conductance of all edges and the boundary conditions at the inlets and outlets of the network. Due to the mass conservation, we can use the Kirchhoff law to determine flow rate $Q_{i j}$ on the edges and the pressure $P_{i}$ at the vertices

$$
\begin{aligned}
& \sum_{j \in N_{i}} Q_{i j}=s_{i}, \\
& Q_{i j}=\left(P_{i}-P_{j}\right) C_{i j},
\end{aligned}
$$

where the lowercase subscripts $i$ and $j$ are used for network vertices - e.g. inlets, outlets, bifurcation points, $N_{i}$ is the neighbour set of the vertex $i, s_{i}$ the given flow source (sink) at the vertex $i, Q_{i j}=-Q_{j i}$ the flow on the edge $\{i, j\}$, and $C_{i j}=C_{j i}$ the conductance of edge 
$\{i, j\}$. In particular, if two nodes $i$ and $j$ are not connected, both $Q_{i j}$ and $C_{i j}$ are zero. We can reformulate the Eqs. (2.3) and (2.4) as

$$
\sum_{j \neq i}\left(P_{i}-P_{j}\right) C_{i j}=s_{i}
$$

or

$$
A \mathbf{P}=\mathbf{s},
$$

where $\mathbf{P}=\left(P_{1}, P_{2}, \ldots, P_{N}\right)^{T}, \mathbf{s}=\left(s_{1}, s_{2}, \ldots, s_{N}\right)^{T}, N$ is the total number of vertices and the coefficient matrix $A$ has the form

$$
A=\left[\begin{array}{cccc}
\sum_{j \neq 1} C_{1 j} & -C_{12} & \ldots & -C_{1 N} \\
-C_{21} & \sum_{j \neq 2} C_{2 j} & \ldots & -C_{2 N} \\
\ldots & \cdots & \cdots & \ldots \\
-C_{N 1} & -C_{N 2} & \cdots & \sum_{j \neq N} C_{N j}
\end{array}\right] .
$$

Note that $-A$ is the graph Laplacian operator of the corresponding weighted graph with the weights are given by the conductances. The Eq. (2.5) is a positive (semi) definite equation set for the pressure at all vertices. Once the boundary conditions - e.g. the pressure or flow at the inlets and outlets are given, the solution can be determined. The Eq. (2.1) can be then used to calculate the wall shear stresses.

\section{Murray's Law}

As was already mentioned, the Murray law is widely observed in different animals [71]. At a bifurcation point of the vessel network, it is observed that the three radii $D, D_{l}$, and $D_{r}$ of the parent vessel and its left and right daughter vessels, respectively, approximately satisfy the cubic relation

$$
D^{3}=D_{l}^{3}+D_{r}^{3}
$$

Murray provided an insightful explanation for this experimental observation - viz. the energy cost for a vessel segment consists of two terms

$$
E_{0}=\Delta P Q+c_{m} V=\frac{128 \mu L Q^{2}}{\pi D^{4}}+\frac{\pi c_{m}}{4} D^{2} L,
$$

where $c_{m}$ is a metabolic constant. The term $\triangle P Q$ is the energy cost to drive the blood flow and $c_{m} V$ the metabolic energy cost for the production and function of the blood in the vessel.

Note that the blood flow $Q$ is demanded by the downstream tissue and may be regarded as a constant. Any discount of the blood flow can lead to dysfunction of the downstream tissue. Minimising the total energy cost $E_{0}$ with respect to the vessel diameter $D$, we obtain the optimised energy cost

$$
E_{m}=\min E_{0}=c_{1} Q^{\frac{2}{3}} L
$$


with the diameter

$$
Q=c_{2} D^{3},
$$

where

$$
c_{1}=\frac{\pi}{4} c_{m} c_{2}^{-\frac{2}{3}}, \quad c_{2}=\frac{\pi}{32} \sqrt{\frac{c_{m}}{\mu}} .
$$

Interestingly, in order to satisfy the optimality condition (2.1), an optimal shear stress need is required uniformly

$$
\tau_{e}=\frac{32 \mu Q}{\pi D^{3}}=\sqrt{\mu c_{m}} .
$$

Note that the flow rate $Q$ in the parent vessel is equal to the sum of the flow rate in the two daughter vessels - i.e.

$$
Q=Q_{l}+Q_{r} .
$$

Substituting the optimality condition (2.1) into the above equation leads to the Murray law (3.1).

Murray's theory initiated various studies of the network structure of circulation systems and stimulated the researches on adaptation dynamics based on mechanical stresses such as the wall shear stress.

\section{Optimisation of General Biological Transport Networks}

\subsection{Optimisation frameworks}

There are different mathematical models for optimisation of general biological transport networks. Here, we discuss the equivalence of three optimisation frameworks used in previous models. This can help us to understand the relations between the models introduced in previous studies.

\subsubsection{Framework 1}

According to Murray arguments, the total energy cost for a biological transport network is

$$
E_{1}(\tilde{\mathbf{C}})=\sum_{\{i, j\}}\left(\frac{Q_{i j}^{2}}{\tilde{C}_{i j}}+c_{m} \tilde{C}_{i j}^{\gamma}\right) L_{i j},
$$

where $\tilde{C}_{i j}=C_{i j} / L_{i j}$ is the conductivity of the edge $\{i, j\}$ and $\gamma \in(0,1)$. In particular, for blood vessels $\gamma=1 / 2$. For leaf veins, we have $1 / 2<\gamma<1$ due to the fact that a leaf vein is a bundle of small tubes [23]. In a more general form, the metabolic or material cost function $m(\tilde{C})$ of an edge has to satisfy the following conditions:

$$
m(0)=0, \quad m^{\prime}(\tilde{C})>0, \quad m^{\prime \prime}(\tilde{C})<0 .
$$

We can check that $m(\tilde{C})=c_{m} \tilde{C}^{\gamma}$ satisfies these conditions if $\gamma \in(0,1)$. 
The flow rates $Q_{i j}$ are determined by the Kirchhoff law (2.3), (2.4). For given flow sources, we can see that the solution $Q_{i j}$ is determined by the conductance vector $\tilde{\mathbf{C}}$, which appears in the coefficient matrix $A$. Therefore, the total energy cost is a function of $\tilde{\mathbf{C}}$. Noting that $\tilde{C}_{i j}=\tilde{C}_{j i}$ and $Q_{i j}=-Q_{j i}$, we compute the gradient of the total energy cost with respect to $\tilde{C}_{k l}$, viz.

$$
\begin{aligned}
\frac{\partial E_{1}(\tilde{\mathbf{C}})}{\partial \tilde{C}_{k l}} & =2\left(c_{m} \gamma \tilde{C}_{k l}^{\gamma-1}-\frac{Q_{k l}^{2}}{\tilde{C}_{k l}^{2}}\right) L_{k l}+2 \sum_{\{i, j\}} \frac{Q_{i j} L_{i j}}{\tilde{C}_{i j}} \frac{\partial Q_{i j}}{\partial \tilde{C}_{k l}} \\
& =2\left(c_{m} \gamma \tilde{C}_{k l}^{\gamma-1}-\frac{Q_{k l}^{2}}{\tilde{C}_{k l}^{2}}\right) L_{k l}+2 \sum_{\{i, j\}}\left(P_{i}-P_{j}\right) \frac{\partial Q_{i j}}{\partial \tilde{C}_{k l}} \\
& =2\left(c_{m} \gamma \tilde{C}_{k l}^{\gamma-1}-\frac{Q_{k l}^{2}}{\tilde{C}_{k l}^{2}}\right) L_{k l}+4 \sum_{\{i, j\}} P_{i} \frac{\partial Q_{i j}}{\partial \tilde{C}_{k l}} \\
& =2\left(c_{m} \gamma \tilde{C}_{k l}^{\gamma-1}-\frac{Q_{k l}^{2}}{\tilde{C}_{k l}^{2}}\right) L_{k l}+4 \sum_{i} P_{i} \frac{\partial \sum_{j \in N_{i}} Q_{i j}}{\partial \tilde{C}_{k l}} \\
& =2\left(c_{m} \gamma \tilde{C}_{k l}^{\gamma-1}-\frac{Q_{k l}^{2}}{\tilde{C}_{k l}^{2}}\right) L_{k l} .
\end{aligned}
$$

It is worth noting that the gradient depends only on local information. For optimised states, we have

$$
Q_{k l}=\frac{1}{\sqrt{c_{m} \gamma}} \tilde{C}_{k l}^{\frac{\gamma+1}{2}} .
$$

This is a generalised form of the cubic relation (3.2).

In the previous studies $[5,6,9,14,15,29]$, the metabolic energy cost is not considered as a part of the energy cost. Instead, a constraint is applied on the total metabolic cost viz. the sum of $\tilde{C}_{i j}^{\gamma}$ is regarded as a constant and a nonlocal Lagrangian multiplier is added to the Lagrangian gradient.

\subsubsection{Framework 2}

In the second framework, the total energy cost

$$
E_{2}(\mathbf{Q}, \tilde{\mathbf{C}})=\sum_{\{i, j\}}\left(\frac{Q_{i j}^{2}}{\tilde{C}_{i j}}+c_{m} \tilde{C}_{i j}^{\gamma}\right) L_{i j}
$$

is optimised under the flow source constraints (2.3), but the Olhm law (2.4) is omitted. Although the total energy cost is the same as in Framework $1, \mathbf{Q}$ and $\tilde{\mathbf{C}}$ are now viewed as independent variables of $E_{2}$. Clearly, the Lagrangian is

$$
L_{2}(\mathbf{Q}, \tilde{\mathbf{C}})=E_{2}(\mathbf{Q}, \tilde{\mathbf{C}})-\sum_{i} \lambda_{i}\left(\sum_{j \in N_{i}} Q_{i j}-s_{i}\right) .
$$


Noting that $Q_{i j}=-Q_{j i}$ in general, we obtain the gradient of the Lagrangian

$$
\begin{aligned}
& \frac{\partial L_{2}(\mathbf{Q}, \tilde{\mathbf{C}})}{\partial Q_{k l}}=2 \frac{Q_{k l}}{C_{k l}}-\lambda_{k}+\lambda_{l}, \\
& \frac{\partial L_{2}(\mathbf{Q}, \tilde{\mathbf{C}})}{\partial \tilde{C}_{k l}}=2\left(c_{m} \gamma \tilde{C}_{i j}^{\gamma-1}-\frac{Q_{k l}^{2}}{\tilde{C}_{k l}^{2}}\right) L_{k l} .
\end{aligned}
$$

With given conductances, the Olhm law (2.4) is spontaneously achieved by optimising $L_{2}(\mathbf{Q}, \tilde{\mathbf{C}})$ with respect to $\mathbf{Q}$. The pressure $P_{k}=\lambda_{k} / 2$ is simply given by the Lagrangian multipliers. The optimisation based on Framework 1 can be considered as the alternating direction method based on Framework 2.

\subsubsection{Framework 3}

Using the optimal condition (4.1) at the critical points, the total energy cost can be written in the form

$$
E_{3}(\mathbf{Q})=\sum_{\{i, j\}} c_{3} Q_{i j}^{\Gamma} L_{i j},
$$

where $\Gamma=2 \gamma /(1+\gamma) \in(0,1)$. The energy cost is also optimised under the flow source constraint (2.3). In this case, the conductance is invisible in the model. We can regard the optimisation of conductance in response to the change of flow rate to be extremely fast. Therefore, the conductance is always given by the optimal condition (4.1).

As far as the alternating direction optimisation in Framework 2 is concerned, the optimisation on the Q-direction leads to the Eq. (2.5), which is incorporated implicitly in Framework 1 and the optimisation on the $\tilde{\mathbf{C}}$-direction leads to the Eq. (4.1), which is incorporated implicitly in Framework 3. In fact, the alternating direction iterations based on the Eqs. (2.5) and (4.1) can be used to find optimal structures of transport networks. From this point of view, the optimal network structures remain the same under the three frameworks. However, due to the nonconvexity in the total energy cost, there exists a variety of optimal structures. Nevertheless, different frameworks generate different optimisation processes and numerical algorithms. Optimisation processes based on the three frameworks may produce different optimal network structures even when they start from the same initial configuration.

Although optimal structures stay the same within the above optimisation frameworks, the constraints under different frameworks, they can be generalised in a different way. The first framework can be generalised to describe systems with diameter-dependent conductances and general relations between the metabolic-cost and conductance - e.g. the Non-Newtonian effect in blood flow is significant when the vessel diameter is close to the diameter of red blood cells. In this case, the effective viscosity is diameter-dependent. However, the constraint of the Ohm law means that the flow is driven by an effective pressure. In the second framework, there is no requirement of the Ohm law. Thus it can be used in the study of systems with more general flow fields. Nevertheless, the conductance idea is 
still important. Since there is no conductance in the third framework, this allows the most genera extensions. The only requirement in this framework is the mass conservation.

\subsection{Topology of optimal biological transport networks}

Using the total energy cost, we can study the topology of those biological transport networks, which have a treelike network skeleton and maintains loop-forming bridge edges between the branches.

\subsubsection{Optimal structure for fixed flow sources}

It has been proved that for fixed flow source constraint, the optimal structure is a loopless treelike network under Framework 1 [15]. We can employ the reduction to absurdity to prove this by using the cost function (4.2) and the flow constraint (2.3). The proof relies on the concavity of the total energy cost under perturbations. It is slightly different from the corresponding proof [15].

Let $\bar{Q}_{i j}$ be optimal flow rates satisfying the flow constraint (2.3). Assume that there is a loop with $K$ vertices $i_{1}, i_{2}, \ldots, i_{K}$ in the optimal network - viz. $\bar{Q}_{i_{m} i_{m+1}} \neq 0$ for $m=$ $1,2, \ldots, K$ and $i_{K+1}=i_{1}$. We add an circulation flow $x$ in the loop

$$
Q_{i_{m} i_{m+1}}(x)=\bar{Q}_{i_{m} i_{m+1}}+x,
$$

while keeping the flow rate on other edges unchanged. It is easily seen that the flow constraint (2.3) is still satisfied. After changing the flow rate, the change of total energy cost is

$$
\Delta E_{3}(x)=\sum_{m=1}^{K} c_{3}\left(Q_{i_{m} i_{m+1}}^{\Gamma}(x)-\bar{Q}_{i_{m} i_{m+1}}^{\Gamma}\right) L_{i j} .
$$

According to our assumption, on any edge of the loop the flow rate $\bar{Q}_{i_{m} i_{m+1}}$ is not equal to zero. Thus we have $\left.\frac{d^{2} \Delta E_{3}(x)}{d x^{2}}\right|_{x=0}<0$ for $\Gamma \in(0,1)$, which is inconsistent with the optimality assumption. Therefore, for fixed flow sources, the optimal network structure is a loopless tree. In fact, $\Delta E_{3}(x)$ becomes non-differentiable and attains a local minimum if and only if the additional circulation flow $x$ cancels the flow on an edge of the loop.

Optimal network structures obtained in our numerical simulations are shown in Fig. 1. If $\gamma>1$, the optimisation problem is rigorously convex and there is only one optimal solution with a large number of small loops. When $\gamma$ decreases, the loop density also decreases slightly. A first order phase transition in loop density can be observed at $\gamma=1$ in the optimal structures. For $\gamma=1$, the optimisation problem is convex but not rigorously convex. As the result, there can be multiple optimal structures. As evidence, we see that the optimal structure in Fig. 1(c) is slightly asymmetric. For $\gamma<1$, the optimal network structure becomes a loopless tree structure. Note that the energy function becomes non-convex in this case. There are a huge amount of optimal structures (local minima), each of which corresponds to a tree-like network structure. 


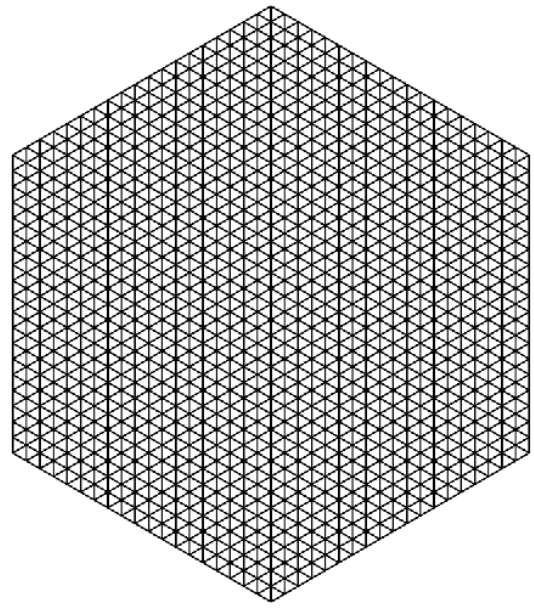

(a)

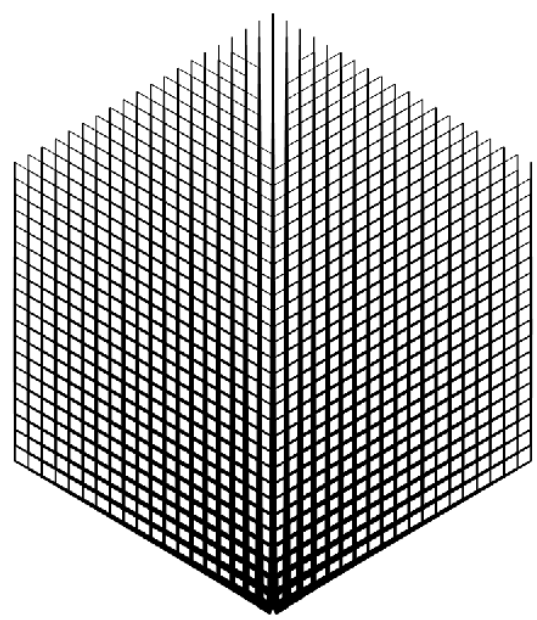

(c)

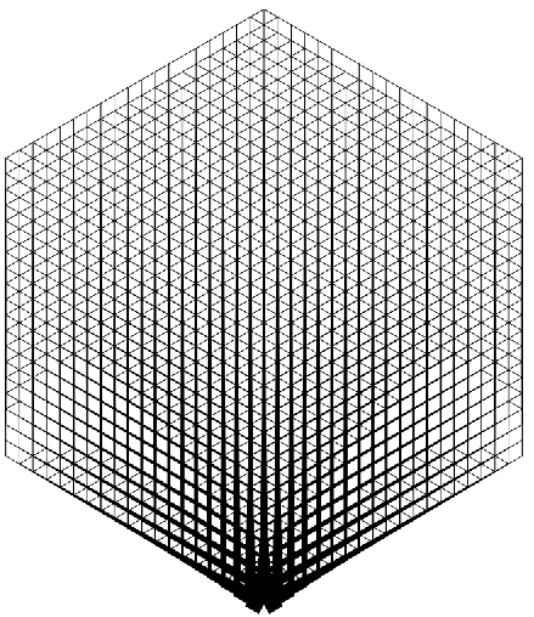

(b)

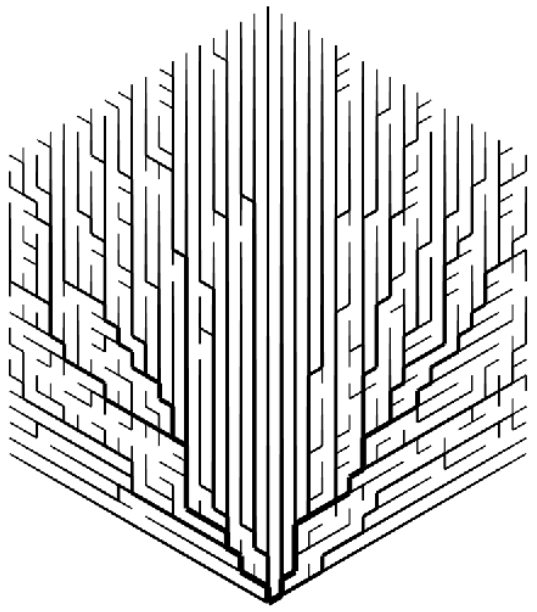

(d)

Figure 1: Optimal structures for fixed source-sinks. The bottom node is set to be the single source node and a unit sink is set on all other nodes. (a) The initial geometry. (b)-(d) The optimal structures for $\gamma=2.0, \gamma=1.0$, and $\gamma=0.5$, respectively. The widths of edges are proportional to $\tilde{C}^{1 / 3}$. The initial conductivities are given as $1+r$, where the random perturbation $r$ is uniformly distributed in $[-0.5,0.5]$.

\subsubsection{Loops in biological transport networks}

Loops exist widely in different transport networks. Theoretical studies show multiple advantages of loopy structures, such as resilience to damage [29] and optimisation of energy cost under fluctuating flow demands $[9,23,29]$. Here we only review in detail the optimisation of network structures under fluctuating flow demands.

Fluctuating flow demands are observed in different biological transport networks, including the open-close switch of stomata [29], the flow regulation of capillary flows [25], and the fluctuating contraction and dilation of the cell membrane of Slime Mold P. poly- 
cephalum [70]. Such fluctuating flow demands have been modeled as a moving sink [29] or random sinks $[9,23]$. In general, we can regard the sink, $s_{i}, i=2,3, \ldots, N$, in the Eq. (2.4) as a random variable (the flow source at the root node $s_{1}=-\sum_{i=2}^{N} s_{i}$ ). Although the neighboring sinks can be correlated [18] for the sake of simplicity we assume that they are uncorrelated. Naturally, the total energy cost function becomes

$$
E_{1}(\tilde{\mathbf{C}})=\sum_{\{i, j\}}\left(\frac{\left\langle Q_{i j}^{2}\right\rangle}{\tilde{C}_{i j}}+c_{m} \tilde{C}_{i j}^{\gamma}\right) L_{i j}
$$

The second moment of flow rate can be evaluated by

$$
\left\langle Q_{i j}^{2}\right\rangle=\left\langle\left(\sum_{k=2}^{N} s_{k} Q_{i j, k}\right)^{2}\right\rangle=\sum_{k=2}^{N} \sigma_{k}^{2} Q_{i j, k}^{2}+\left(\sum_{k=2}^{N} e_{k} Q_{i j, k}\right)^{2},
$$

where $e_{k}=-\left\langle s_{k}\right\rangle$ and $\sigma_{k}^{2}$ are the expectation and variance of the random flow sinks at sink $k$, whereas $Q_{i j, k}$ is the flow in edge $\{i, j\}$ at the single-sink state that only $s_{1}=-s_{k}=1$ are nonzero. In particular, when the random sinks are independent identically distributed (i.i.d.), we have

$$
\left\langle Q_{i j}^{2}\right\rangle=\sigma^{2} \sum_{k=2}^{N} Q_{i j, k}^{2}+e^{2} \bar{Q}_{i}^{2},
$$

where $\bar{Q}_{i}=\sum_{k=2}^{N} Q_{i j, k}$.

In what follows, we only consider i.i.d. random sinks. In this case, we can use dimensional arguments to show that the topology of the optimal network structure depends on the ratio $\sigma / e$ only. Since the open-close switch for the sinks is observed in different biological transport networks, uniform open probability $p$ and uniform strength $1 / \sqrt{P}$ are introduced for all sinks - cf. [25]. More exactly, each sink has a probability $p$ to be open independently with $s=1 / \sqrt{P}$, whereas $s=0$ when the sink is closed. In this case, $\sigma / e=\sqrt{(1-p) / p}$, and the second moment of flow rate is

$$
\left\langle Q_{i j}^{2}\right\rangle=(1-p) \sum_{k=2}^{N} Q_{i j, k}^{2}+p \bar{Q}_{i}^{2}
$$

The open probability can be used to quantitatively describe the strength of fluctuation. Thus a greater open probability $p$ means a smaller fluctuation in flow rate. For $p=1$, the fixed sink case reemerges. For $p \rightarrow 0$, the moving-sink case is obtained in [29].

Optimal network structures obtained with fluctuating flow sinks (sources) are shown in Fig. 2. We observe that even if $\gamma<1$, there are numerous loops in the optimal transport network when fluctuation in flow is sufficiently strong (sufficiently small $p$ ). The loop number is an increasing function of $\gamma$ and a decreasing function of $p$. For fixed $\gamma$, as the open probability increases, the number of loops decreases and reaches zero at a sufficiently large $p$. For $\gamma=0.25$ and $p=0.5$ the optimal structure does not contain any loop. 

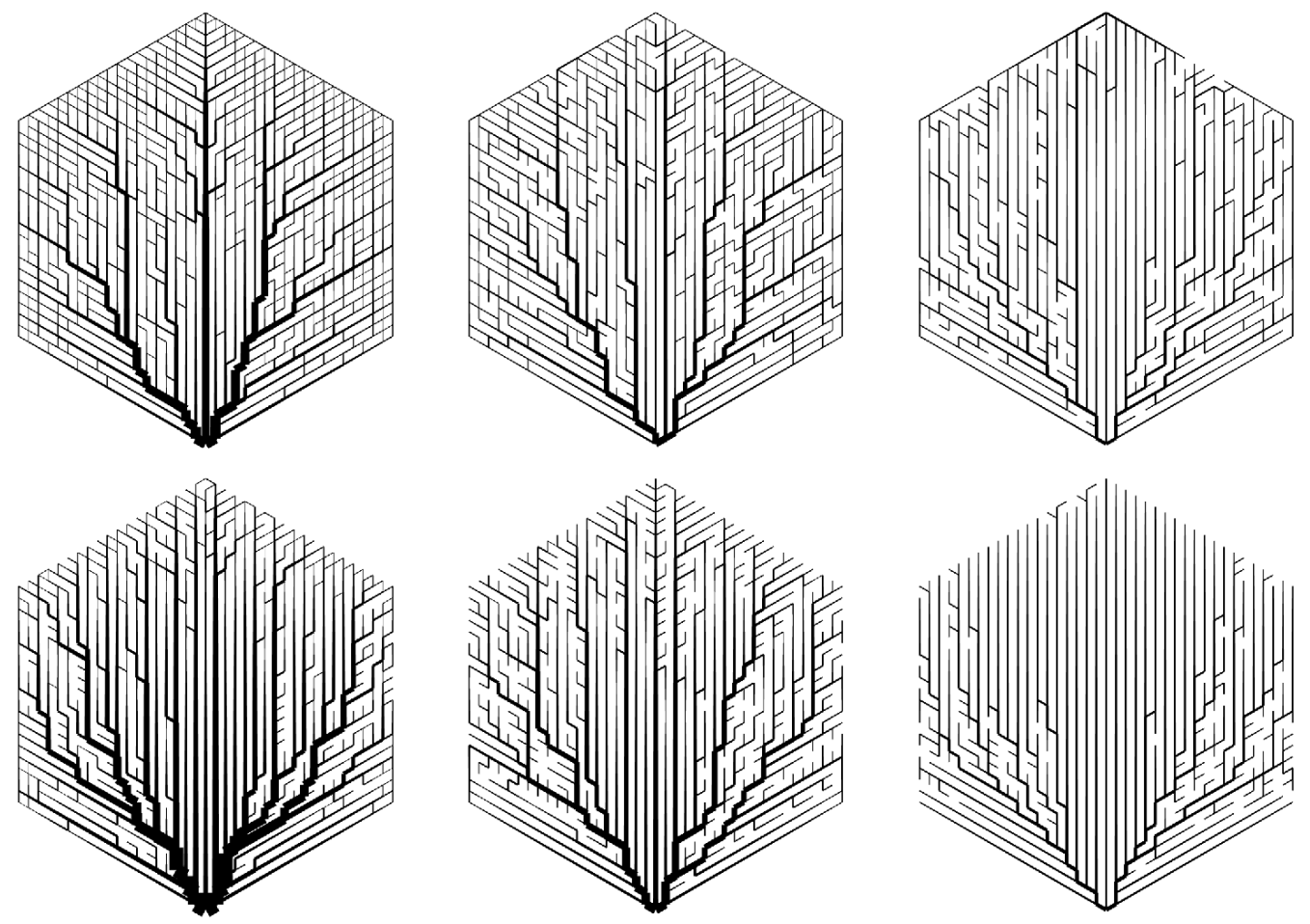

Figure 2: Optimal structures for fluctuating sinks. The bottom node is set to be the single source node and all other nodes are endowed with random sinks with open probability $p$. The upper panel: $p=0.1$; The bottom panel: $p=0.5$. The left-, the middle-, and the right-panels correspond to $\gamma=0.75, \gamma=0.5$, and $\gamma=0.25$, respectively. Other settings are as same as in Fig. 1.

Similar to the network formed by the Slime Mold P. polycephalum, any node can be a source or sink node at random and $\left\langle s_{k}\right\rangle=0, \sum_{k=1}^{N} s_{k}=0$. In this case, we have

$$
\left\langle Q_{i j}^{2}\right\rangle=\left\langle\left(\sum_{k=1}^{N}\left(s_{k}-\frac{1}{N} \sum_{l=1}^{N} s_{l}\right) Q_{i j, k}\right)^{2}\right\rangle=\sum_{k=1}^{N} \sigma_{k}^{2}\left(Q_{i, k}-\frac{1}{N} \bar{Q}_{i}\right)^{2}
$$

The corresponding optimal structures embedded in a two dimensional plane are shown in Fig. 3. We note that for the variance $\sigma_{k}^{2}$ uniform for all nodes (a)-(c), the optimal structure is a decentralised network. In each of these networks, all nodes maintain a similar degree of connections to other nodes. For $\gamma>1$, there are numerous long range connections and it is not convenient to show the network in a figure. For $\gamma=1$, the optimal network appears to be well clustered locally. As $\gamma$ decreases, the number of loops becomes smaller and smaller. In the limit case, the network becomes loopless and maintains a relatively small total length. It is interesting that we can effectively control the total number of edges (or the degree of each node) by selecting suitable $\gamma$. When the variance is extremely different ((d) in Fig. 3), nodes with large variances become the centers in optimal networks. 


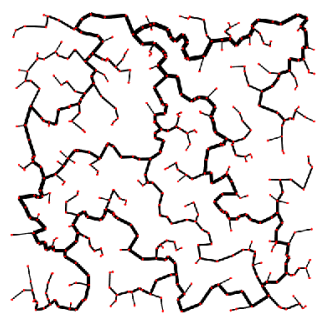

(a) $\gamma=0.1$

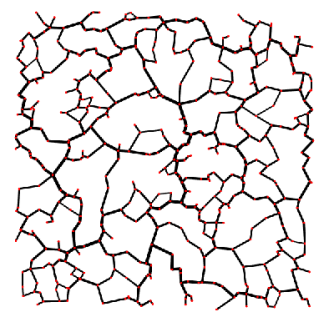

(b) $\gamma=0.5$

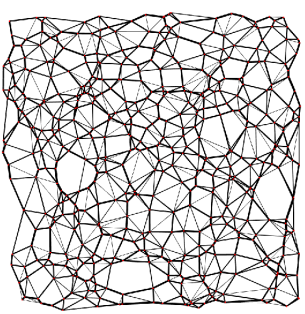

(c) $\gamma=1.0$

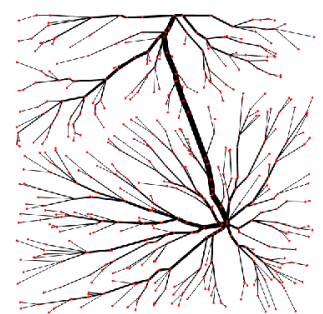

(d) $\gamma=0.5$

Figure 3: Optimal structures for random source-sinks (shown by the red dots). The distance between two nodes is given by their Euclidean distance. An all-to-all network with uniform conductivity 1 is used as the initial value. (a)-(c) Optimal networks for random source-sinks with uniform variances $\sigma_{k}^{2}$. (d) Optimal networks for random source-sinks with extremely nonuniform variances $\sigma_{k}^{2}$.

\subsection{Matrix optimisation and optimal transport networks}

Using the Kirchhoff law (2.5), the energy cost function for random source-sinks with uniform variances can be written in the form

$$
\begin{aligned}
E_{1}(\tilde{\mathbf{C}}) & =\left\langle\mathbf{s}^{T} \mathbf{A}^{-1} \mathbf{s}\right\rangle+c_{m} \sum_{\{i, j\}} \tilde{C}_{i j}^{\gamma} L_{i j} \\
& =\operatorname{tr}\left(\mathbf{A}^{-1} \Sigma\right)+c_{m} \sum_{\{i, j\}} \tilde{C}_{i j}^{\gamma} L_{i j},
\end{aligned}
$$

where $\Sigma$ is the covariance matrix of the sources. In particular, for i.i.d. random flow sinks we have

$$
\begin{aligned}
E_{1}(\tilde{\mathbf{C}}) & =\sigma^{2} \operatorname{tr}\left(\mathbf{A}^{-1}\right)+c_{m} \sum_{\{i, j\}} \tilde{C}_{i j}^{\gamma} L_{i j} \\
& =\sigma^{2} \sum_{i} \lambda_{i}^{-1}+c_{m} \sum_{\{i, j\}} \tilde{C}_{i j}^{\gamma} L_{i j},
\end{aligned}
$$

where $\lambda_{i}, i=1,2, \ldots, N$ are the eigenvalues of $\mathbf{A}$. In this case, network optimisation is closely related to many matrix optimisation problems such as maximisation of the smallest eigenvalue with given constraints or minimisation of the ratio between maximal and minimal eigenvalues $[3,56]$. In this case, the optimised network structure is called "entangled network" $[3,12]$. This kind of network structures usually maintains good robustness.

Eigenvalues obtained from the matrix optimisation problem (4.3) under different geometrical setups are shown in Fig. 4. As $\gamma$ decreases, the number of edges also decreases. As the consequence, the average distance between network nodes $k$ grows, which enlarges the ratio between the maximal and minimal eigenvalues.

\section{Adaptation of General Biological Transport Networks}

Circulation systems of animals are well designed to respond to both short-term and longterm change of tissue demands. The short-term response, called the blood flow regulation, 

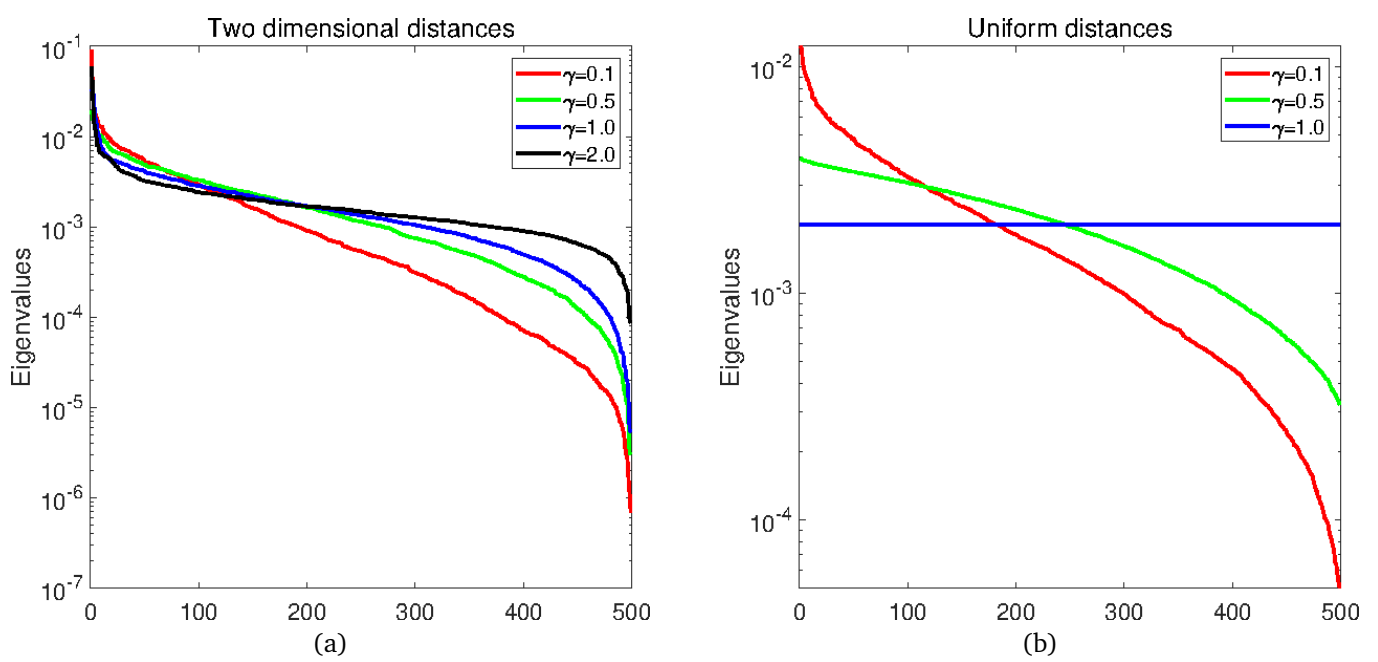

Figure 4: Eigenvalues of the coefficient matrix $A$. (a) The distances $L_{i j}$ are given by the Euclidean distances between the nodes in a plane. (b) The distances $L_{i j}$ of all edges are set to be 1 .

includes multiple means - viz. the blood pressure controlling at the heart, the luminal diameter changes of blood vessels, and the open rate modulation of the capillary flows [13, 31-33,58]. Meanwhile, as the long-term response, the chronic adaptation of the circulation system also involves multiple structural changes including the luminal diameters, the microvessel density, and the vessel wall thicknesses [10,36, 37, 45, 47, 50,67]. By means of the blood flow regulation and the adaptation of blood vessels, the efficiency and robustness of circulation systems are maintained spontaneously. This is closely related to the structural optimisation of the circulation system.

The shear stress on blood vessel walls plays an important role in both short-term and long-term response. For example, as a short-term response, the vessel dilates when the blood flow increases whereas contracts when the blood flow decreases. As the consequence, the change wall shear stress due to flow changes is buffered and it leads to a relatively steady-going level of the wall shear stress [31-33,58]. The endothelial cells of blood vessels are responsible for sensing the wall shear stress. Meanwhile, a long-term change of blood flows also leads to structural changes in the vessel luminal diameter [60]. As the Murray law shows, the wall shear stress is relatively uniform in the entire circulation system in optimal structures. This has been further validated by different experiments $[28,42]$.

Using the experimental observations of [28,31-33,42,58], Hacking et al. [19] and Pries et al. [59] introduced the following minimal model of the blood vessel diameter adaptation:

$$
\frac{d D_{i j}}{d t}=c_{0}\left(\tau_{i j}-\tau_{e}\right) D_{i j}=c_{0}\left(\frac{32 \eta Q_{i j}}{\pi D_{i j}^{3}}-\tau_{e}\right) D_{i j},
$$

where $c_{0}$ is a positive constant corresponding to the growth rate of the diameters. This model describes the balance between an intrinsic decreasing tendency due to cell death and other effects, $-c_{0} \tau_{e} D_{i j}$, and a growth effect $c_{0} \tau_{w i} D_{i j}$ stimulated by the wall shear stress. 


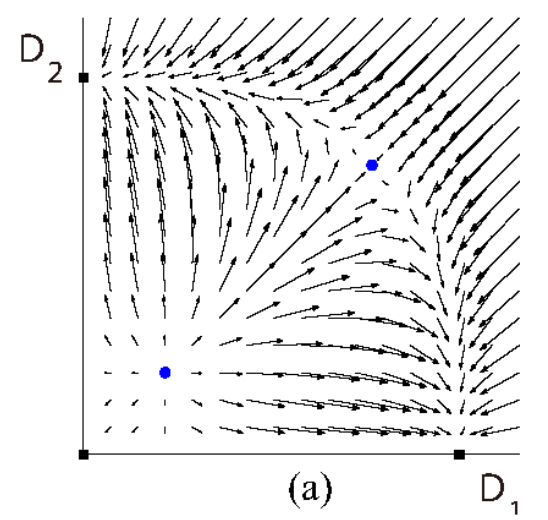

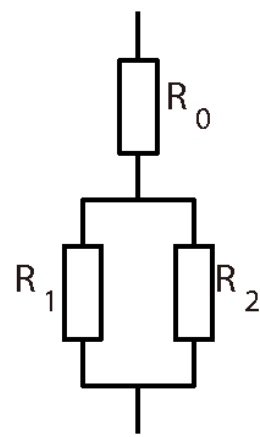

(b)

Figure 5: (a) Phase-plane illustration of the adaptation process of the two parallel vessels. The icons in (b) are borrowed from that used in electric circulation. The two black squares in (a) denote the stable steady states, whereas the two blue dots denote the unstable steady states. An arrow starting at a point $\left(D_{1}, D_{2}\right)$ is used to show the changing rate of the two diameters.

Taking into account the relation between conductance and the vessel diameter (2.2), one can write the adaptation model in the form

$$
\frac{d C_{i j}}{d t}=4 c_{0}\left(\tau_{i j}-\tau_{e}\right) C_{i j}
$$

The most important feature of the adaptation model (5.1) is that the adaptation of in parallel-vessel systems is unstable [19]. As shown in Fig. 5, in the parallel systems there can be at most one survived vessel at the stable states. In Fig. 5(a), we show the adaptation process of the two diameters in a parallel system shown in Fig. 5(b). A fixed pressure drop is maintained in the simulation. The adaptation of two parallel vessels with fixed total flow rates is similar. The instability of parallel systems indicates that it is energetically favorable to deliver the fluid together instead of distributing the fluid into different tubes. This is consistent with the concavity of the energy cost function $E_{3}$ defined in the Eq. (4.2).

In Ref. [23], a model in the form of gradient flow with respect to the total energy cost function $E_{1}(\tilde{\mathbf{C}})$ is introduced to describe the adaptation of general biological transport networks

$$
\frac{d \tilde{C}_{i j}}{d t}=c^{\prime}\left(\frac{\left\langle Q_{i j}^{2}\right\rangle}{\tilde{C}_{i j}^{\gamma+1}}-\tilde{\tau}_{e}^{2}\right) \tilde{C}_{i j},
$$

where $\tilde{\tau}_{e}^{2}=c_{m} \mu$ is the optimal wall shear stress (3.3). Note that the new form can be used to naturally incorporate the fluctuation of flow rates into the model. In the sense of linearisation, the behavior of this model is very similar to that of the adaptation model (5.1). Thus the corresponding experimental observations are also consistent with this gradient flow model. Optimal network structures are obtained at steady states as time tends to infinity. From this point of view, the steady states shown in Fig. 5 are the local minima of the total energy cost function and the adaptation trajectories are the gradient flows. This 
new model clearly shows that the adaptation process is a tool employed by life systems to optimise the structure of transport networks.

\section{Continuum Models of Initiation of Biological Transport Networks}

Although the adaptation processes can lead to optimisation of biological transport networks, the emergence of transport networks in life systems is also of a particular interest. As was already noted, there are different cell-based models, which emphasize the importance of such cellular mechanisms as cell migration and elongation. In the canalisation hypothesis, the importance of auxin transport has been singled out as the stimulus in the emergence of leaf veins. However, the importance of structural optimisation during the network initiation has hardly been explored in these models.

Despite the migration and reuse of the endothelial cells of pruned vessels [8], initially optimised biological transport networks may prevent frequent vessel pruning in vasculogenesis or angiogenesis processes. From this point of view, the cellular pathways, which can incorporate structural optimisation during the initiation process of biological transport networks, can significantly reduce the cost of the energy needed toe construct blood vessel systems. For plants, their vascular cells cannot move freely. As the result, for plants the pruning processes are even more energy-costly than for animals. Therefore, the initial optimisation of transport networks is more important for plants.

In Ref. [24], a continuum model has been constructed as a general macroscopic principle underlying the cellular mechanisms in the initiation process. In this model, it is conjectured that the total effective energy cost function is optimised during the initiation process of biological transport networks.

Since transport processes are usually much faster than initiation ones, the transport process of auxin and other mass can be described by the steady state diffusion equation

$$
\nabla \cdot(\mathbf{A}(\mathbf{x}, \mathbf{t}) \cdot \nabla P(\mathbf{x}, t))=s,
$$

where $P$ is the partial pressure generated by hormone - e.g. auxin, $s$ the hormone source produced in the tissue, and $\mathbf{x}$ and $t$ are the spatial and temporal coordinates. The transport tensor A is used to account pure diffusion effects, active transport, and pipe flow in veins. The discretisation of the Eq. (6.1) is similar to discretisation of the Eq. (2.5). Adaptation models for the transport tensor are introduced below. According to the restricted form of A, continuum adaptation models are called scalar-, vector-, and tensor-based. The three models have similar energy-cost functionals and all of them can lead to the formation of optimal transport networks, though their dynamic behavior are slightly different.

\subsection{Vector based model}

As experimentally observed $[4,11,48,69]$, active transport of auxin in leaf budding is localised at specific paths. Transmembrane proteins are lined up along specific directions, which determine the transport path. Following such observations, one describes the enhancement of active transport by polarisation vector $\mathbf{m}$. The direction of $\mathbf{m}$ stands for the 
direction of active transport whereas its amplitude $|\mathbf{m}|$ quantifies the transport strength. Consequently, the transport tensor is modeled as

$$
\mathbf{A}=D \mathbf{I}+\tilde{\mathbf{A}}=D \mathbf{I}+\mathbf{m} \otimes \mathbf{m},
$$

where $D$ is the diffusion constant of the background substrate, I the identity tensor, and $\otimes$ the tensor product. If $|\mathbf{m}|$ is sufficiently large - e.g. it exceeds a critical value $m_{0}$, the vein of the transport network is formed. In this case, $|\mathbf{m}|^{2}$ represents the conductivity of the tube per unit area.

The total energy cost functional has the form

$$
E=\int_{\Omega}\left(\langle\nabla P \cdot \mathbf{A} \cdot \nabla P\rangle+M\left(|\mathbf{m}|^{2}\right)+\alpha|\nabla \mathbf{m}|^{2}\right) d x
$$

where $\Omega$ is the tissue domain and $x$ the spatial coordinate. The term $\langle\nabla P \cdot \mathbf{A} \cdot \nabla P\rangle$ represents the average energy cost in mass transport for all different states with fluctuating fluxes and $M\left(|\mathbf{m}|^{2}\right)$ shows the material and metabolic energy cost of maintaining the active transport or constructing the transport network edges. This function is usually assumed to be concave for the formation of vascular segments, and very often one uses the power function $M(C)=$ $c_{m} C^{\gamma}$. We also note that $\alpha|\nabla \mathbf{m}|^{2}$ is the entropy cost associated with the diffusion of proteins responsible for active transport.

The adaptation dynamics optimising the energy cost functional can be modeled by the equation

$$
\frac{\partial \mathbf{m}}{\partial t}=D_{0} \Delta \mathbf{m}+c_{0}\left(\langle(\mathbf{m} \cdot \nabla P) \nabla P\rangle-M^{\prime}\left(|\mathbf{m}|^{2}\right) \mathbf{m}\right),
$$

where $c_{0}$ determines the time scale of the initiation process and $D_{0}=2 c_{0} \alpha$ is the diffusion constant of the polarisation signal. The process is dominated by two factors competition viz. the polarisation of cells driven by hormone fluxes to enhance the delivery power and the background contracting tendency to reduce the material and metabolic cost.

The energy cost functional $E$ for the coupled system (6.1)-(6.2) satisfies the estimate

$$
\frac{d E}{d t}=-\frac{1}{c_{0}} \int_{\Omega}\left(D_{0} \triangle \mathbf{m}+c_{0}\left(\langle(\mathbf{m} \cdot \nabla P) \nabla P\rangle-M^{\prime}\left(|\mathbf{m}|^{2}\right) \mathbf{m}\right)\right)^{2} d x \leq 0 .
$$

This inequality implies that the energy consumption of the system decreases continuously. As time increases, the state of the system approaches a stability point where an optimised structure is obtained.

\subsection{Scalar-based model}

If transport power is isotropic instead of direction-specific, one can use $\tilde{\mathbf{A}}=\tilde{A} \mathbf{I}$ to model the enhancement of the transport power. In this case, an adaptation dynamics of $\tilde{A}$ satisfies the equation

$$
\frac{\partial \tilde{A}}{\partial t}=D_{0} \triangle \tilde{A}+c_{0}\left(\langle\nabla P \cdot \nabla P\rangle-M^{\prime}(\tilde{A})\right),
$$


which can also lead the system to an optimal structure due to the following relation for the energy cost functional:

$$
\frac{d E}{d t}=-\frac{1}{c_{0}} \int_{\Omega}\left(D_{0} \triangle \tilde{A}+c_{0}\left(\left\langle|\nabla P|^{2}\right\rangle-M^{\prime}(\tilde{A})\right)\right)^{2} d x .
$$

\section{Tensor-based model}

The adaptation dynamics can be also described by the polarisation tensor $\tilde{\mathrm{A}}$ by the equation

$$
\frac{\partial \tilde{\mathbf{A}}}{\partial t}=D_{0} \triangle \tilde{\mathbf{A}}+c_{0}\left(\langle\nabla P \otimes \nabla P\rangle-M^{\prime}(|\tilde{\mathbf{A}}|) \frac{\tilde{\mathbf{A}}}{|\tilde{\mathbf{A}}|}\right),
$$

where $|\tilde{\mathbf{A}}|$ is the Frobenius norm of Ã. Similarly, the energy cost functional satisfies

$$
\frac{d}{d t}=-\frac{1}{c_{1}} \int_{\Omega}\left|D_{0} \triangle \tilde{\mathbf{A}}+c_{1}\left(\langle\nabla P \otimes \nabla P\rangle-M^{\prime}(|\tilde{\mathbf{A}}|) \frac{\tilde{\mathbf{A}}}{|\tilde{\mathbf{A}}|}\right)\right|^{2} d x .
$$

In the canalisation model [11], the authors consider the critical strength of active transport. Above this critical strength, the cells are assumed to undergo cell differentiation to form vessel segments. In continuum models, this idea is represented by the dual role of the polarised transport tensor $|\mathbf{A}|-$ viz. if the magnitude $|\mathbf{A}|$ is greater than some critical value $A_{0}$, the signal is strong enough and a vascular segment is formed at the principal direction of $|\mathbf{A}|$. In this case, the integral of $|\mathbf{A}|$ in a cross-section can be regarded as the conductivity of the vascular segment. Furthermore, after the discretisation, the Eq. (6.1) can be viewed as the Kirchoff laws (2.5) for flow distributions in the network, while (6.2)(6.4) are effectively reduced to the adaptation process of the segments (5.2), cf. $[8,23,66]$. In other words, the initiation and adaptation processes are integrated into a single model. The domain $|\mathbf{A}|>A_{0}$ forms a network-like structure, which can be regarded as the space occupied by the biological transport network.

Fig. 6 shows that continuum models exhibit rich dynamics, which leads to the formation of a plethora of optimal structures. Note that for a fixed source distribution $s$, loopless tree-like structures are obtained at steady states due to the concavity of the metabolic and material cost function $M$. Besides, because of background diffusion in the transport equation (6.1), the bifurcation of branches ends at a particular length scale, below which the pure diffusion is sufficiently effective to undertake the transportation tasks. The diffusion term in the Eqs. (6.2)-(6.4) introduces another length scale corresponding to the width of the vascular segments. If both diffusion constants vanish in a limiting case, the bifurcation never ends and a fractal structure is obtained.

Fig. 6 demonstrates that the network obtained by a tensor-based model appears to be more regular than the one obtained by the vector-based model. This is due to the fact that the polarisation direction changes more easily in the tensor based model. Similarly, in the isotropic scalar-based model, the tree-like structure can be even more regular because of the absence of polarisation directions. Fig. 6(a) contains fuzzy areas, which arose because of the long time required to approach steady state in vector-based models. 


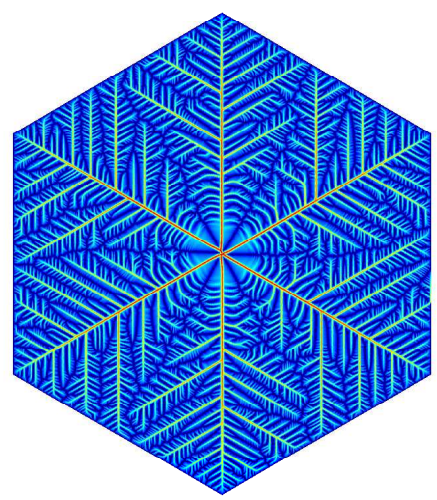

(a)

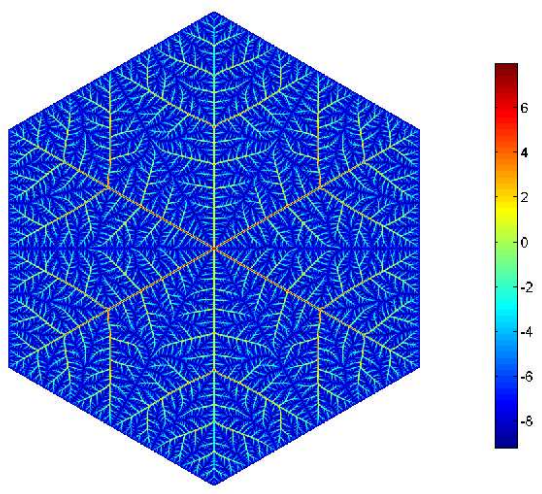

(b)

Figure 6: Optimal tree-like network structures obtained with (a) the vector-based model and (b) the tensor-based model. Colors are used to indicate the amplitude of $\log \left(|\tilde{\mathbf{A}}|^{2}+D\right)$. A constant source $\left(s=s_{0}\right)$ is uniformly distributed in the entire domain and a single sink is located at the center. The variance of the random initial value of the polarisation vector is given by the diffusion constant $\sqrt{D}$. The Neumann boundary conditions are used in the simulation for both Eqs. (6.1) and (6.2). Parameters: $D=1 \times 10^{-3}$, $\alpha=1 \times 10^{-6}, c_{0}=5 \times 10^{-3}, b=1, s_{0}=1, \gamma=0.5$, and hexagon edge length is 1 .

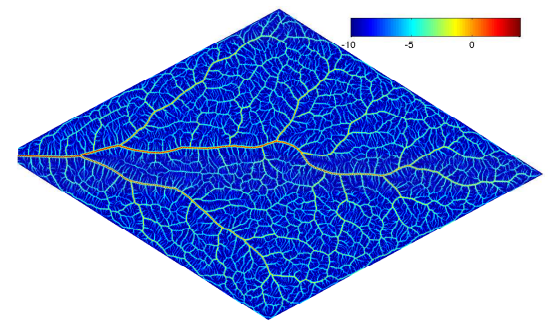

(a)

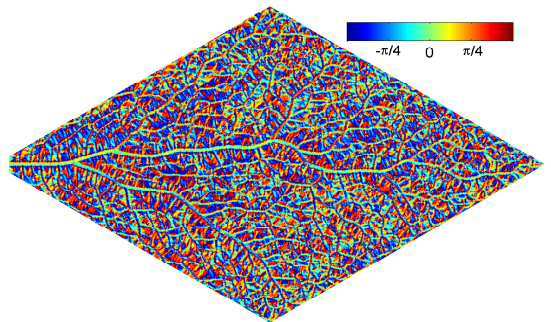

(b)

Figure 7: An optimal loopy network obtained with the vector-based model with fluctuating sources. (a) Magnitude of the polarisation vector. (b) Angle of the polarisation direction, which shows that the polarisation vector on an edge is approximately tangential to the edge. The entire domain is divided into 900 small sub-domains. At each simulation step, sources on only 10 sub-domains are randomly selected to be open. The Dirichlet boundary condition is applied for the Eq. (6.1) on the short left edge. The Neumann boundary conditions are used for other boundaries and the adaptation equation. Parameters: $D=1 \times 10^{-6}, \alpha=1 \times 10^{-6}, c_{0}=5 \times 10^{-3}, b=1, s_{0}=1$, and $\gamma=0.5$. The length of the diamond edge is 1 .

In the case of fluctuations in the production of hormone source $s$, there are loops in the networks formed by the initiation dynamics - cf. Fig. 7. The loop density is still an increasing function of the fluctuation strength and a decreasing function of $\gamma$.

\section{Conclusion and Discussion}

We have reviewed the development history on modeling and theoretical studies of biological transport networks. Following Murray's theoretical work, studies on biological transport network have been carried out along two paths - one emphasizes optimisation 
of energy cost and the other focuses on adaptation mechanism and relevant stimuli. These approaches merge into a single model by incorporating the gradient flow of the total energy cost in the adaptation dynamics and the optimal network structures are obtained at the steady states of the adaptation dynamics.

It is worth noting that the adaptation governed by the gradient flow with local stimuli is in great consistency with experimental observations. It has been successfully employed to explain the vessel pruning in embryol zebra fish [8]. The canalisation mechanism driven by the gradient flow is also qualitatively consistent with experimental observations of the leaf venation formations. In other words, under the stress of natural selection, life systems develop various ways to optimise the total energy consumption for mass transportation. For plants, the optimisation of the network structure is mainly achieved in the initiation process since the cells cannot move freely. For animals, the structural optimisation is mainly achieved by the adaptation process - endothelial cells of the pruned vessels can be reused to form new vessels.

Nevertheless, there is a large amount of loops in the capillary bed. Current models still cannot explain the stability of many loops. Meanwhile, the efficiency of capillary networks is very crucial for animals. It remains a mystery how the angiogenesis and adaptation process lead to stable and high-efficiency capillary networks. Note that only macroscopic signals such as the wall shear stress in blood vessels are involved in the macroscopic dynamics, whereas the cellular dynamics, which achieves such an adaptation is masked. The optimisation principle should also provide critical insights in the complete adaptation model for the entire circulation system.

The beautiful mathematical structures of the models for biological transport networks have attracted widespread interest. The nice robustness and high efficiency of optimal transport networks can have wide applications in industrial and biomimetic network design $[27,46]$, such as three-D printed vascular networks.

\section{Acknowledgments}

This work is supported by the National Key R\&D Program of China (2019YFA0709503), the National Natural Science Foundation of China (Contract No. 11971312), and by the Student Innovation Center, Shanghai Jiao Tong University.

\section{References}

[1] G. Albi, M. Artina, M. Foransier and P.A. Markowich, Biological transportation networks: Modeling and simulation, Anal. Appl. 14, 185-206 (2016).

[2] G. Albi, M. Burger, J. Haskovec, P. Markowich and M. Schlottbom, Continuum modeling of biological network formation, in: Active Particles 1, pp. 1-48, Springer (2017).

[3] A. Arenas, A. Díaz-Guilera, J. Kurths, Y. Moreno and C. Zhou, Synchronization in complex networks, Phys. Rep. 469, 93-153 (2008).

[4] O. Avsian-Kretchmer, J. Cheng, L. Chen, E. Moctezuma and Z.R. Sung, Indole acetic acid distribution coincides with vascular differentiation pattern during arabidopsis leaf ontogeny, Plant Physiol. 130, 199-209 (2002). 
[5] J.R. Banavar, F. Colaiori, A. Flammini, A. Maritan and A. Rinaldo, Topology of the fittest transportation network, Phys. Rev. Lett. 84, 4745 (2000).

[6] S. Bohn and M.O. Magnasco, Structure, scaling and phase transition in the optimal transport network, Phys. Rev. Lett. 98, 088702 (2007).

[7] H. Chen, H. Leng, D. Wang and X. Wang, An efficient threshold dynamics method for topology optimization for fluids, ArXiv:1812.09437 (2018).

[8] Q. Chen, L. Jiang, C. Li, D. Hu, Ji. Bu, D. Cai and J. Du, Haemodynamics-driven developmental pruning of brain vasculature in zebrafish, PLoS Biol. 10, e1001374 (2012).

[9] F. Corson, Fluctuations and redundancy in optimal transport networks, Phys. Rev. Lett. 104, 048703 (2010).

[10] D. Desplanches, Structural and functional adaptations of skeletal muscle to weightlessness, Int. J. Sports Med. 18, S259-S264 (1997).

[11] P. Dimitrov and S.W. Zucker, A constant production hypothesis guides leaf venation patterning, Proc. Natl. Acad. Sci. U.S.A. 103, 9363-9368 (2006).

[12] L. Donetti, P.I. Hurtado and M.A. Munoz, Entangled networks, synchronization and optimal network topology, Phys. Rev. Lett. 95, 188701 (2005).

[13] B.R. Duling, Microvascular responses to alterations in oxygen tension, Circ. Res. 31, 481-489 (1972).

[14] M. Durand, Architecture of optimal transport networks, Phys. Rev. E 73, 016116 (2006).

[15] M. Durand, Structure of optimal transport networks subject to a global constraint, Phys. Rev. Lett. 98, 088701 (2007).

[16] D. Fang, S. Jin, P. Markowich and B. Perthame, Implicit and semi-implicit numerical schemes for the gradient flow of the formation of biological transport networks, SMAI J. Comput. Math. 5, 229-249 (2019).

[17] A. Gaelle, R. Lagan and P. Prusinkiewicz, Reviewing models of auxin canalization in the context of leaf vein pattern formation in arabidopsis, Plant J. 44, 854-865 (2005).

[18] J. Grawer, H. Ronellenfitsch, M.G. Mazza and E. Katifori, Trophallaxis-inspired model for distributed transport between randomly interacting agents, Phys. Rev. E 96, 022111 (2017).

[19] W.J. Hacking, E. VanBavel and J.A. Spaan, Shear stress is not sufficient to control growth of vascular networks: a model study, Am. J. Physiol-Heart C. 270, H364-H375 (1996).

[20] J. Haskovec, L.M. Kreusser and P. Markowich, Rigorous continuum limit for the discrete network formation problem, Commun. Partial Differ. Equations 44, 1159-1185 (2019).

[21] J. Haskovec, P. Markowich and B. Perthame, Mathematical analysis of a pde system for biological network formation, Commun. Partial Differ. Equations 40, 918-956, (2015).

[22] Q. Hong, J. Zhao and Q. Wang, Energy-production-rate preserving numerical approximations to network generating partial differential equations, Comput. Math. Appl. 84, 148-165 (2021).

[23] D. Hu and D. Cai, Adaptation and optimization of biological transport networks, Phys. Rev. Lett. 111, 138701 (2013).

[24] D. Hu and D. Cai, An optimization principle for initiation and adaptation of biological transport networks, Commun. Math. Sci. 17, 1427-1436 (2019).

[25] D. Hu, D. Cai and A.V. Rangan, Blood vessel adaptation with fluctuations in capillary flow distribution, PloS One 7, e45444 (2012).

[26] E.A. Jones, F. le Noble and A. Eichmann, What determines blood vessel structure? genetic prespecification vs. hemodynamics, Physiology 21, 388-395 (2006).

[27] F. Kaiser, H. Ronellenfitsch and D. Witthaut, Discontinuous transition to loop formation in optimal supply networks, Nat. Commun. 11, 1-11 (2020).

[28] A. Kamiya, R. Bukhari and T. Togawa, Adaptive regulation of wall shear stress optimizing vascular tree function, Bull. Math. Biol. 46, 127-137 (1984). 
[29] E. Katifori, G.J. Szollosi and M.O. Magnasco, Damage and fluctuations induce loops in optimal transport networks, Phys. Rev. Lett. 104, 048704 (2010).

[30] J.B. Kirkegaard and K. Sneppen, Optimal transport flows for distributed production networks, Phys. Rev. Lett. 124, 208101 (2020).

[31] A. Koller and G. Kaley, Endothelial regulation of wall shear stress and blood flow in skeletal muscle microcirculation, Am. J. Physiol-Heart C. 260, H862-H868 (1991).

[32] A. Koller, D. Sun and G. Kaley, Role of shear stress and endothelial prostaglandins in flow-and viscosity-induced dilation of arterioles in vitro, Circ. Res. 72, 1276-1284 (1993).

[33] L. Kuo, W.M. Chilian and M.J. Davis, Interaction of pressure-and flow-induced responses in porcine coronary resistance vessels, Am. J. Physiol-Heart C. 261, H1706-H1715 (1991).

[34] M.F. Laguna, S. Bohn and E.A. Jagla. The role of elastic stresses on leaf venation morphogenesis, PLoS Comput. Biol. 4, e1000055 (2008).

[35] L. Lamalice, F.L. Boeuf and J. Huot, Endothelial cell migration during angiogenesis, Circ. Res. 100, 782-794 (2007).

[36] J.M. Lash and H.G. Bohlen, Functional adaptations of rat skeletal muscle arterioles to aerobic exercise training, J. Appl. Physiol. 72, 2052-2062 (1992).

[37] A.M. Lewis, O. Mathieu-Costello, P.J. McMillan and R.D. Gilbert. Effects of long-term, highaltitude hypoxia on the capillarity of the ovine fetal heart, Am. J. Physiol-Heart C. 277, H756H762 (1999).

[38] B. Li, Long time behavior of the solution to a parabolic-elliptic system, Comput. Math. Appl. 78, 3345-3362 (2019).

[39] B. Li, On the blow-up criterion and global existence of a nonlinear pde system in biological transport networks, Kinet. Relat. Mod. 12, 1131 (2019).

[40] B. Li and X. Li, A cross-diffusive evolution system arising from biological transport networks, Commun. Nonlinear Sci. Numer. Simul. 92, 105465 (2021).

[41] B. Li and J. Shen, Classical solution of a pde system stemming from auxin transport model for leaf venation, Proc. Am. Math. Soc. 148, 2565-2578 (2020).

[42] H.H. Lipowsky and B.W. Zweifach, Network analysis of microcirculation of cat mesentery, Microvasc. Res. 7, 73-83 (1974).

[43] J. Liu and X. Xu, Partial regularity of weak solutions to a pde system with cubic nonlinearity, J. Differ. Equations 264, 5489-5526 (2018).

[44] Y. Lu, D. Hu and W. Ying, A fast numerical method for oxygen supply in tissue with complex blood vessel network, PloS One 16, e0247641 (2021).

[45] C. Lundby, M. Sander, G.V. Hall, B. Saltin and J.A. Calbet, Maximal exercise and muscle oxygen extraction in acclimatizing lowlanders and high altitude natives, J. Physiol. 573, 535-547 (2006).

[46] M. Mao, H. Bei, C.H. Lam, P. Chen, S. Wang, Y. Chen, J. He and X. Zhao, Human-on-leafchip: A biomimetic vascular system integrated with chamber-specific organs, Small 16, 2000546 (2020).

[47] O. Mathieu-Costello and P.J. Agey, Chronic hypoxia affects capillary density and geometry in pigeon pectoralis muscle, Resp. Physiol. 109, 39-52 (1997).

[48] J. Mattsson, Z.R. Sung and T. Berleth, Responses of plant vascular systems to auxin transport inhibition, Development 126, 2979-2991 (1999).

[49] K.A. McCulloh, J.S. Sperry and F.R. Adler, Water transport in plants obeys Murray's law, Nature 421, 939-942 (2003).

[50] K.S. McDonald, M.D. Delp and R.H. Fitts, Effect of hindlimb unweighting on tissue blood flow in the rat, J. Appl. Physiol. 72, 2210-2218 (1992).

[51] R.M. Merks, S.V. Brodsky, M.S. Goligorksy, S.A. Newman and J.A. Glazier, Cell elongation is 
key to in silico replication of in vitro vasculogenesis and subsequent remodeling, Dev. Biol. 289, 44-54 (2006).

[52] G.J. Mitchison, A model for vein formation in higher plants, P. Roy. Soc. Lond. B. Bio. 207, 79-109 (1980).

[53] G.J. Mitchison, The polar transport of auxin and vein patterns in plants, Philos. Trans. R. Soc. London, Ser. B 295, 461-471 (1981).

[54] C.D. Murray, The physiological principle of minimum work: I. the vascular system and the cost of blood volume, Proc. Natl. Acad. Sci. U.S.A. 12, 207 (1926).

[55] T. Nelson and N. Dengler, Leaf vascular pattern formation, The Plant Cell 9, 1121 (1997).

[56] T. Nishikawa, A.E. Motter, Y. Lai and F.C. Hoppensteadt, Heterogeneity in oscillator networks: Are smaller worlds easier to synchronize, Phys. Rev. Lett. 91, 014101 (2003).

[57] T.V. Oevelen and M. Baelmans, Numerical topology optimization of heat sinks, in: International Heat Transfer Conference Digital Library, Begel House Inc. (2014).

[58] U. Pohl, J. Holtz, R. Busse and E. Bassenge, Crucial role of endothelium in the vasodilator response to increased flow in vivo, Hypertension 8, 37-44 (1986).

[59] A.R. Pries, B. Reglin and T.W. Secomb, Structural adaptation of vascular networks: role of the pressure response, Hypertension 38, 1476-1479 (2001).

[60] A.R. Pries and T.W. Secomb, Control of blood vessel structure: insights from theoretical models, Am. J. Physiol-Heart C. 288, H1010-H1015 (2005).

[61] H. Ronellenfitsch and E. Katifori, Global optimization, local adaptation and the role of growth in distribution networks, Phys. Rev. Lett. 117, 138301 (2016).

[62] H. Ronellenfitsch and E. Katifori, Phenotypes of vascular flow networks, Phys. Rev. Lett. 123, 248101 (2019).

[63] T. Sachs, The control of the patterned differentiation of vascular tissues, Adv. Bot. Res. 9, 151262 (1981).

[64] T. Sachs, The development of vascular networks during leaf development, Curr. Top. Plant Biochem. Physiol. 8, 168-183 (1989).

[65] T. Sachs, Collective specification of cellular development, BioEssays 25, 897-903 (2003).

[66] T. Secomb, J.P. Alberding, R. Hsu, M.W. Dewhirst and A.R. Pries, Angiogenesis: an adaptive dynamic biological patterning problem, PLoS Comput. Biol. 9, e1002983 (2013).

[67] T.V. Serebrovskaya, E.B. Manukhina, M.L. Smith, H.F. Downey and R.T. Mallet, Intermittent hypoxia: cause of or therapy for systemic hypertension, Exp. Biol. Med. 233, 627-650 (2008).

[68] J. Shen and B. Li, A priori estimates for a nonlinear system with some essential symmetrical structures, Symmetry 11, 852 (2019).

[69] L.E. Sieburth, Auxin is required for leaf vein pattern in arabidopsis, Plant Physiol. 121, 11791190 (1999).

[70] A. Tero, S. Takagi, T. Saigusa, K. Ito, D.P. Bebber, M.D. Fricker, K. Yumiki, R. Kobayashi and T. Nakagaki, Rules for biologically inspired adaptive network design, Science 327, 439-442 (2010).

[71] R. Thoma, Untersuchungen über die Histogenese und Histomechanik des Gefässsystems, Enke (1893).

[72] X. Xu, Partial regularity of weak solutions and life-span of smooth solutions to a biological network formulation model, SN Partial Differ. Equations Appl. 1, 1-31 (2020). 